\title{
Spheroid formation and modulation of tenocyte-specific gene expression under simulated microgravity
}

\author{
Armin Kraus \\ Ronald Luetzenberg \\ Nauras Abuagela \\ Siri Hollenberg \\ Manfred Infanger
}

Department of Plastic, Aesthetic and Hand Surgery, Otto-von-Guericke University, Magdeburg, Germany

Corrisponding Author:

Armin Kraus

Department of Plastic, Aesthetic and Hand Surgery,

Otto-von-Guericke University

Leipziger Strasse 44

D-39120 Magdeburg, Germany

E-mail: armin.kraus@med.ovgu.de

\section{Summary}

Background: For tendon tissue engineering, tenocyte-seeded scaffolds are a promising approach. Under conventional 2D culture however, tenocytes show rapid senescene and phenotype loss. We hypothesized that phenotype loss could be counteracted by simulated microgravity conditions.

Methods: Human tenocytes were exposed to microgravity for 9 days on a Random Positioning Machine (RPM). Formation of 3D-structures (spheroids) was observed under light microscopy, gene expression was measured by realtime PCR. Cells under conventional 2D-culture served as control group.

Results: Simulated microgravity reached a value

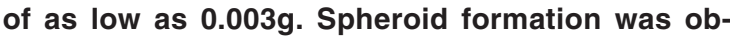
served after 4 days, and spheroids showed stable existance to the end of the observation period. After 9 days, spheroids showed a significantly higher gene expression of collagen 1 (Col1A1) compared to adherent cells under microgravity $(4.4 x$, $\mathrm{p}=0.04)$ and compared to the control group $(5.6 \mathrm{x}$, $\mathrm{p}=0.02)$. Gene expression of collagen 3 (COL3A1) was significantly increased in spheroids compared to the control group (2.3x, $p=0.03)$. Gene expressions of the extracellular matrix genes Tenascin $C$ und Fibronectin (TNC and FN) were increased in adherent cells under microgravity compared to the $1 \mathrm{~g}$-control group, not reaching statistical significance $(p=0.1$ and $p=0.3)$. For the gene expression of vimentin, no significant alteration was observed both in the adherent cells and in the spheroids compared to the $1 \mathrm{~g}$ control group. Gene expression of the tenocyte-specific transcription factor scleraxis (SCX) was significantly increased in spheroids compared to the control group (3.7x, $\mathrm{p}=0.03)$.

Conclusion: Simulated microgravity could counteract tenocyte senescence in vitro and serve as a promising model for scaffold-free 3D cell culturing and tissue engineering.

Level of evidence: V (laboratory study).

KEY WORDS: microgravity, cell senescence, tenocytes, tendon, tissue engineering.

\section{Introduction}

In complex hand injuries, tendon grafting is often required. However, the number of suitable autologous donor tendons is limited, so that there is a demand for tissue engineered tendon grafts. These contructs consist of an acellular scaffold providing mechanical stability, and a seeded cell line providing regeneration and tissue function. Several scaffold materials have been used with some success so far, such as chitosan, silk fibroin, PLGA or decellularized cadaver tendons ${ }^{1-4}$. Compared to other cell lines such as skin fibroblast and adipose stem cells ${ }^{5,6}$, tenocytes seem highly suitable or scaffold seeding as the most original cell line ${ }^{7}$. However, tenocytes are difficult to obtain in sufficient number, and when expanded in vitro, they quickly lose their original phenotype. Yao et al. ${ }^{4}$ observed changes in morphology and rapid decrease in cell proliferation, collagen 1 production and decorin expression during $2 \mathrm{D}$ in vitro culture after as few as four passages. Attempts with 3D culture models have been undertaken to overcome this problem of in vitro senescence. Stoll et al. ${ }^{8}$ could show higher expression of tendon related genes such as collagen 1, scleraxis, aggrecan, COMP and decorin in both highdensity cultures of tenocytes and in a PLGA matrix compared to conventional 2D culture.

As both culture in artificial matrices and high-density cultures are difficult to handle technically, it was our aim to establish a 3D culture model by use of simulated microgravity. Simulated microgravity has been shown to induce three-dimensional cell formation (spheroids) in various cell types, such as thyroid can- 
cer cells ${ }^{9}$, corneal stroma cells ${ }^{10}$ or endothelial cells ${ }^{11}$. We therefore hypothesized that spheroid formation will also take place in human tenocytes exposed to microgravity and that spheroid formation will counteract in vitro senescence in analogy to the 3D culture models mentioned above.

\section{Methods}

\section{Cell culture}

Specimens of human tendons were obtained from patients undergoing hand surgery. Tissue that had to be resected and would have been discarded otherwise was collected. Written consent was obtained from the patients prior to surgery and the procedure was approved by the institutional review board.

The Authors meet the ethical standards of this journal as described by Padulo et al. previously ${ }^{12}$.

Tendon samples from four patients were used for tenocyte cultivation, cells were pooled in the experiments in order to compensate for interindividual differences that could falsify the results. Tendon specimens were minced into $1 \mathrm{~mm}$ pieces in a sterile fashion and placed into cell culture tubes. Sterile trypsine (Sigma Aldrich, $\mathrm{MO}$ ) was added in a volume 5 times the one of the tendon sample and samples were incubated at $37^{\circ} \mathrm{C}$ for 15 minutes. The suspension was centrifuges at 2000rpm for 5 minutes and the supernatant was removed. Tendon fragments were then incubated in collagenase type I (Sigma) at a concentration of $5 \mathrm{mg} / \mathrm{ml}$ in phosphatebuffered saline (PBS) for 1 hour. The suspension was then passed through a sterile cell strainer to remove debris and the filtrate was centrifuged at 2000rpm for 15 minutes. The remaining cell pellet was resuspended in culture medium (Ham's F12 with 10\% fetal bovine serum, both from Invitrogen, MO). For the experiments, tenocytes of passage 4 were used, as an expansion until $4^{\text {th }}$ passage was necessary to obtain a sufficient cell number. During the culture period, no alteration of cell morphology or growth speed had been observed neither in this experiment nor in our prior studies with tenocytes.

\section{Random positioning machine (RPM)}

The exact function of the RPM used in this study has been described in detail elsewhere ${ }^{13}$. Briefly, an incubator had been installed on rotating frames. The incubator is rotated at constant velocity, but the rotation direction is inverted at random time points, averaging the gravity vector. Good correlation of microgravity simulated by this method was found to microgravity in space experiments ${ }^{14,15}$.

5 T25 culture flasks were seeded with $5 \times 10^{5}$ human tenocytes respectively and incubated over night at $37^{\circ} \mathrm{C}$ and $5 \% \mathrm{CO}_{2}$ in a standard incubator to ensure attachment of the cells to the bottom of the culture flask. The next day, the flasks were completely filled with medium carefully avoiding air bubbles. 5 T25 flasks were fixed on the RPM, as close as possible to the center of the platform, which was then rotated at a speed of $60 \%$ in real random mode. The RPM was positioned in a commercially available incubator set at $37^{\circ} \mathrm{C}$ and supplied with $5 \% \mathrm{CO}_{2}$. An equal number of T25 culture flasks were kept in an incubator at $1 \mathrm{~g}$ as control group, at $37^{\circ} \mathrm{C}$ and $5 \% \mathrm{CO}_{2}$. Cell flasks were kept under microgravity conditions for 9 days. Control flask were filled in exactly the same fashion without air bubbles as the flasks in the experimental group. Half of the medium was changed in both the experimental and the control group after 5 days. Interruption of microgravity was kept to a period as short as possible.

\section{Phase contrast microscopy}

Spheroid formation was observed under phase contrast microscopy after 4 and 8 days. Interruption of microgravity conditions for this purpose was limited to a duration as short as possible. The Axiovert $25 \mathrm{Mi}-$ croscope (Carl Zeiss Microscopy, Germany) was used for this purpose.

\section{Real-time PCR}

\section{RNA isolation}

RNA isolation was performed using the RNeasy $\odot$ Mini Kit (Qiagen) according to the manufacturer's recommendations. Cells were detached from the culture wells with $0.25 \%$ trypsine (Sigma). A cell count was performed using a hemocytometer to allow for approximately equal starting number of cells. The cell suspension was spun in an RNase-free tube for 5 minutes at $300 \mathrm{~g}$. The pellet was lysed by adding 350 $\mu \mathrm{l}$ of the lysis buffer (RLT). Cell-inherent RNases were inactivated by adding $1 \% \beta-\mathrm{ME}$. The lysate was homogenized by the QIAshredder ${ }^{\odot}$ system (Qiagen). It was then stabilized by adding 1 volume of $70 \%$ ethanol. DNase (Qiagen, Hilden, Germany) was added in the process of RNA isolation, in order to diminish residual DNA contaminations. The solution was then added to an RNeasy $\odot$ Spin Column and centrifuged in a microcentrifuge at $10,000 \mathrm{rpm}$ for 15 seconds. The flow-through was discarded. Subsequently, $700 \mu$ l of the washing-buffer RW1 was added to the spin column and the column was centrifuged again at $10,000 \mathrm{rpm}$ for 15 seconds, the flow-through was discarded. $500 \mu$ l of the eluting buffer RPE were added to the spin column and centrifuged at 10,000 rpm for 15 seconds. This step was repeated after discarding the flow-through. The spin column was then placed into an RNase-free collection tube and the RNA was eluted with $30 \mu \mathrm{l}$ of RNase-free water. RNA amount was measured using the absorbance at 260 $\mathrm{nm}$. The A260/280 ratio was measured to determine RNA purity. RNA content of the solution was quantified via Photometer Ultrospec2010 (Amersham Biosciences, Freiburg, Germany).

\section{Reverse transcription}

Reverse transcription was performed using the first strand cDNA synthesis kit (ThermoFisher Scientific, Waltham, US), following manufacturer's instructions. Briefly, total cellular RNA was incubated together with 
random hexamer primers, reverse transcriptase, nucleotides, RNase inhibitor in reaction buffer. Temperature profile was $25^{\circ} \mathrm{C}$ for 5 minutes followed by $42^{\circ} \mathrm{C}$ for 60 minutes. The same device was used for creating reverse transcription temperature profile and performing real-time PCR (7500 Fast Real-Time PCR System, Applied Biosystems, Darmstadt, Germany). CDNA solution was stored at $-20^{\circ} \mathrm{C}$ if not used for real-time PCR immediately.

\section{Real-Time PCR}

Real-time PCR was performed with a 7500 Fast RealTime PCR System using Fast SYBR Green PCR Master Mix (both Applied Biosystems). Experimental data was normalized to the input amount of RNA. Primers for collagen I and III, tenascin C, fibronectin, vimentin and scleraxis were identified from literature as indicated in Table I and manufactured by TibMolBiol, Berlin Germany. For scleraxis, a commercially available primer assay was used ( $\mathrm{RT}^{2}$ qPCR Assay for Human SCXB, SABiosciences, sequence not revealed by manufacturer). Primers were synthesized by TIB Molbiol (Berlin, Germany), sequences are shown in Table I. SYBR Green Master Mix (Applied Biosystems) was used for amplification of target cDNA. The following cycling steps were performed: $95^{\circ} \mathrm{C}$ for 10 minutes (enzyme activation), $95^{\circ} \mathrm{C}$ for 15 seconds, $60^{\circ} \mathrm{C}$ for 1 minute (40 cycles). Specific amplification was confirmed by dissociation curves. For calculating $x$-fold changes in gene expression compared to the control, the $2^{\Delta \Delta \mathrm{Ct}}$ method was applied. Differences in the $\mathrm{Ct}$ values were normalized to the expression of $18 \mathrm{~s}$ as a housekeeping gene, $1 \mathrm{~g}$ conditions were set as $100 \%$.

\section{Statistics}

All statistical analyses were performed using SPSS 21.0 (SPSS, Inc., Chicago, IL, USA, 2012). The data was analyzed with the Mann-Whitney $U$ tests. Calcu- ations were performed using the $2^{\wedge}$ delta $\mathrm{Ct}$ values. The data was expressed as means \pm standard deviation (SD). Differences were considered significant at $p<0.05$. The post-hoc power calculation revealed $97 \%$ power to determine a $100 \%$ difference in gene expression for the group number of $n=5$.

\section{Results}

Simulated microgravity reached a value as low as $0.003 \mathrm{~g}$.

\section{Phase contrast microscopy}

After 4 days, formation of tenocyte spheroids consisting of three to five cells was observed, with no obvious impaction of cell viability (Fig. 1 a, b). After 8 days, size of spheroids appeared slightly increased, with a cell number of up to ten per spheroid. All spheroids showed cluster-shaped size. Part of the cells kept attached to the culture vessel under microgravity conditions, regular attachment of the tenocytes to cell culture flask was observed in the control group (Fig. 2 a, b).

\section{Gene expression}

After 9 days, tenocye spheroids showed a significantly higher gene expression of collagen 1 (Col1A1) compared to the $1 \mathrm{~g}$ control group (5.6x, $\mathrm{SD}=1.60$, $\mathrm{p}=0.02)$ and to the adherent cells under microgravity $(4,4 x, S D=1.20, p=0,04$, Fig. 3 a). Gene expression of collagen 3 (COL3A1) was significantly increased in spheroids compared to the control group $(2.3 \mathrm{x}$, $\mathrm{SD}=0.37, p=0.03$, Fig. $3 \mathrm{~b}$ ), but not for the adherent group $(p=0.06)$. Gene expressions of the extracellular matrix genes Tenascin $C$ und Fibronectin (TNC and $F N$ ) were increased in adherent cells under microgravity compared to the $1 \mathrm{~g}$-control group, not reaching statistical significance $(1.5 \mathrm{x}, \mathrm{SD}=0.07$ for tenascin

Table I. Primer sequences.

\begin{tabular}{lll}
\hline Primer & Gene & Source \\
\hline $\begin{array}{l}\text { Fwd 5'TCTCTCCTCTGAAACCCTCCTC3' } \\
\text { Rev 5'GTGCTTTGGGAAGTTGTCTCTG3' }\end{array}$ & Collagen I & \\
Fwd 5'GCTGGCTACTTCTCGCTCTG3' & \\
Rev 5'TTGGCATGGTTCTGGCTTCC3' & Collagen III & \\
Fwd 5'GAGATATGGGGACAATAACCACAG3' & \\
Rev 5'ATTTCTGAAGTTGCTTGGTCTCAG3' & Tenascin C \\
Fwd 5'CCGTGGGCAACTCTGTC3' & \\
Rev 5'TGCGGCAGTTGTCACAG3' & Fibronectin \\
Fwd 5'GACACTATTGGCCGCCTGCAGGATGAG3' & Vimentin \\
Rev 5'ACTGCAGAAAGGCACTTGAAAGC3' & & $(17)$ \\
sequence not revealed by manufacturer & Scleraxis & SCX B RT² qPCR Primer Assay, Qiagen \\
\hline
\end{tabular}




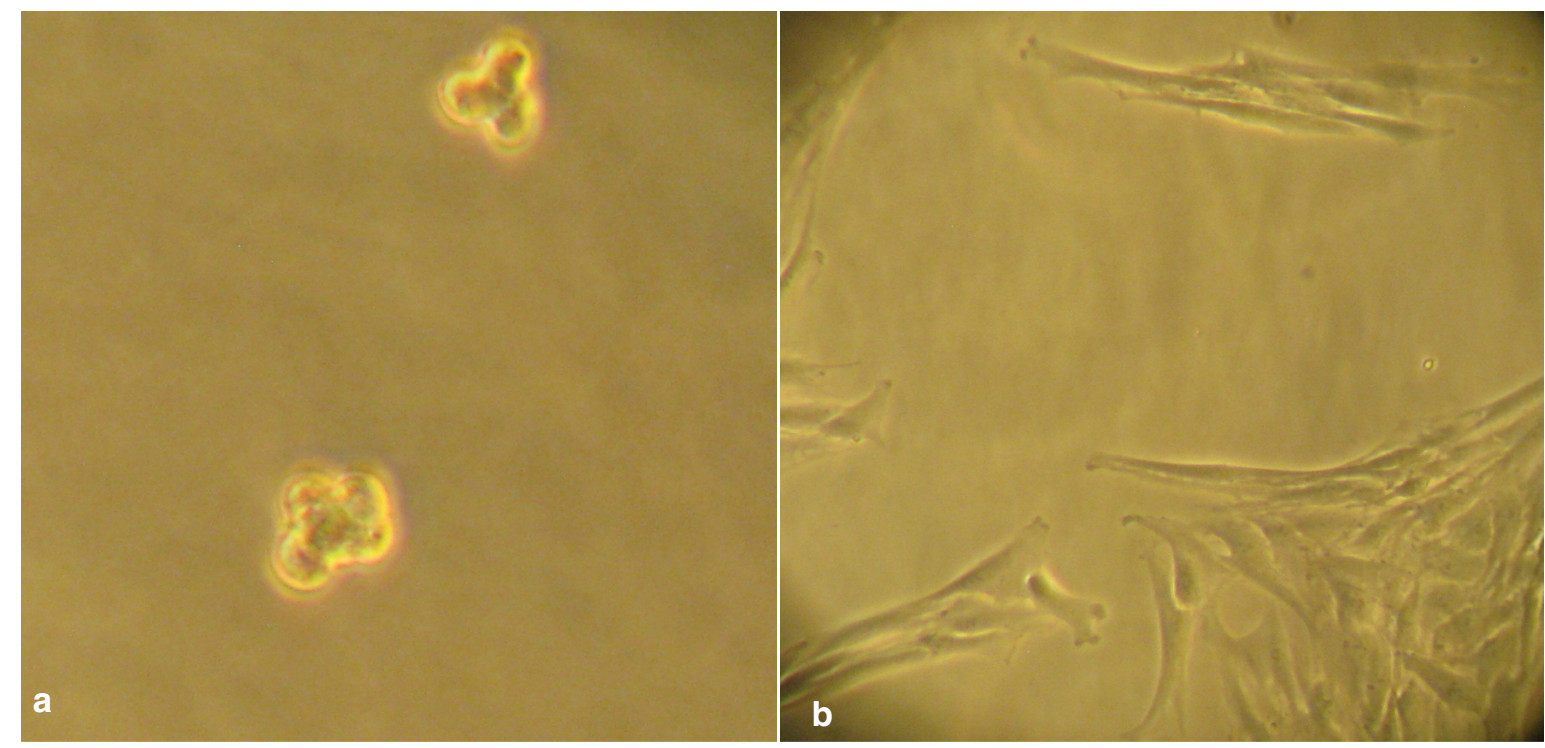

Figure 1 a,b. a. Spheroid formation of tenocytes after four days under microgravity. b. $1 \mathrm{~g}$ control group after 4 days. All cells are attached to the surface.

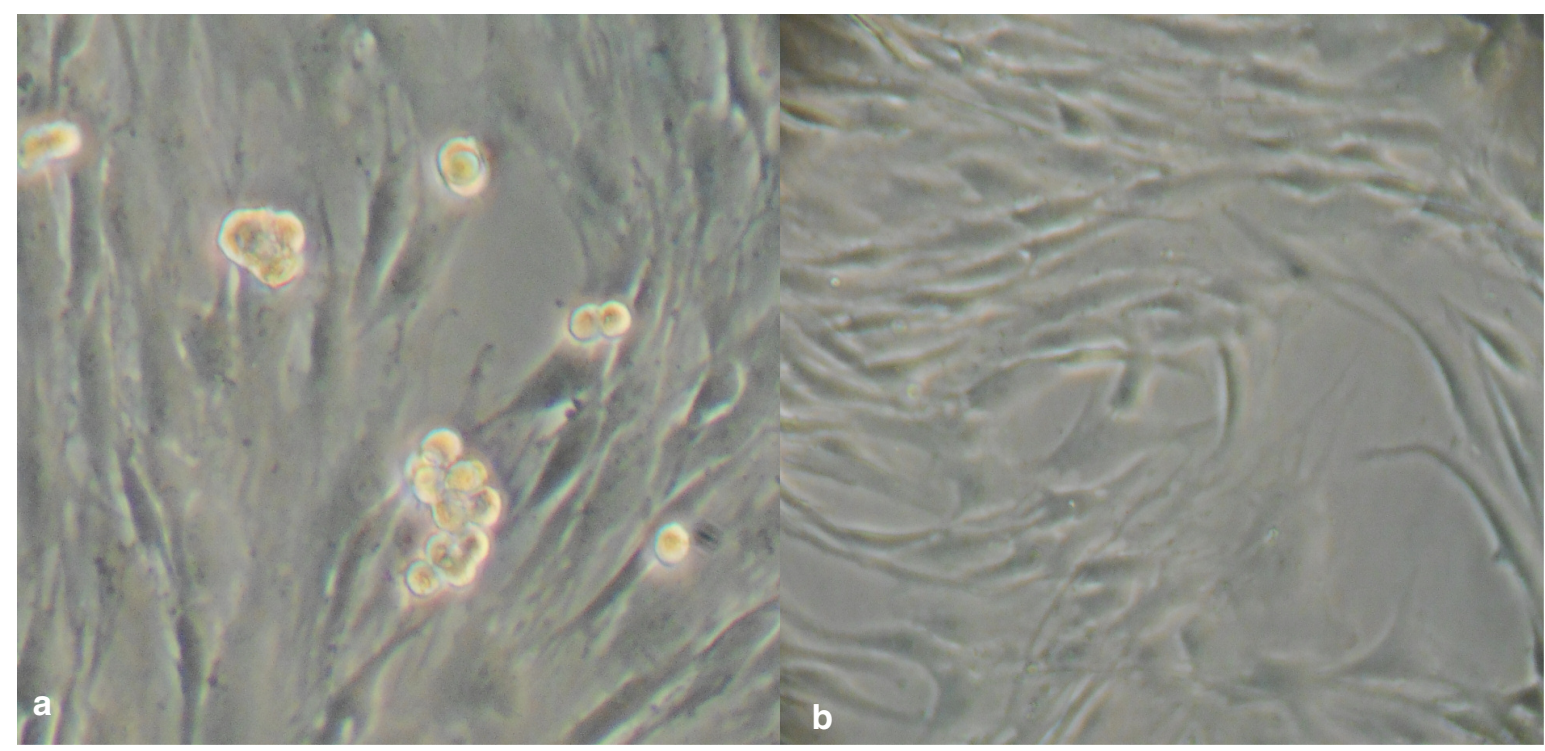

Figure 2 a,b. a. Spheroid formation of tenocytes after 8 days under microgravity, part of the cells still attached to the surface, good cell viability and increase in the size of the spheroids. b. Control group after 8 days - all cells attached to the surface.

C, $p=0.1$, and $2.1 x, S D=0.63$, for fibronectin, $p=0,3$ Fig. $3 \mathrm{c}, \mathrm{d}$ ). For the gene expression of vimentin, no significance alteration was observed both in the adherent cells and in the spheroids compared to the $1 \mathrm{~g}$ control group (Fig. 3 e). Gene expression of the tenocyte-specific transcription factor scleraxis $(S C X)$ was significantly increased in spheroids compared to the control group (3.7x, SD=0.69, p=0.03, Fig. 3 f).

\section{Discussion}

As tenocytes are hard to obtain in a number sufficient for tendon tissue engineering, expansion in vitro is inevitable to the date. Under conventional culture conditions in a dish or flask, cells are plated in 2-dimensional layer, which builts a fairly artificial environment, that has been shown to be detrimental to phenotype preservation in various cell lines. Loss of differentiation potential into osteogenic and adipogenic lineage has been described for adipose-derived stem cells ${ }^{20,21}$ during monolayer culture. On the other hand, by 3D-cultivation in microfibers, human pluripotent stem cells have preserved their undifferentiated phenotype to a significantly higher passage than in culture on a matrigel surface ${ }^{22}$. Similarly, phenotype 

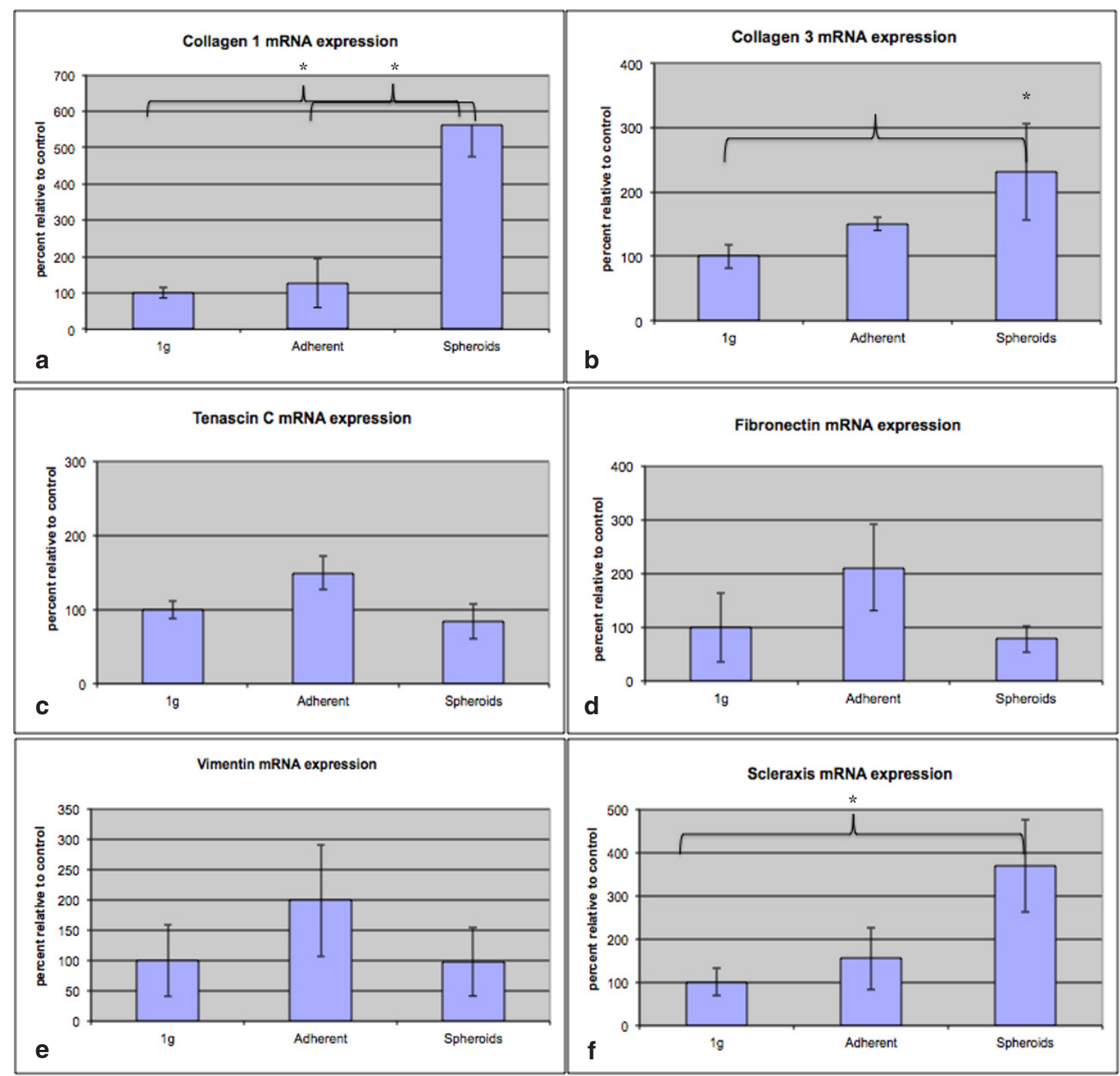

Figure 3 a,b. a. Significantly higher gene expression of collagen 1 (Col1A1) in spheroids compared to adherent cells under microgravity $(4.4 x, p=0.04)$ and compared to the control group (5.6x, $p=0.02)$. b. Gene expression of collagen 3 (COL3A1) was significantly increased in spheroids compared to the control group (2.3x, $S D=0.37, p=0.03$ ). c. Gene expression of the extracellular matrix gene Tenascin $C(T N C)$ was increased in adherent cells under microgravity compared to the $1 \mathrm{~g}$-control group, not reaching statistical significance. d. Gene expression of the extracellular matrix gene $\mathrm{Fi}$ bronectin (FN) was increased in adherent cells under microgravity compared to the $1 \mathrm{~g}$-control group, not reaching statistical significance. e. Gene expression of vimentin: no significant alteration was observed both in the adherent cells and in the spheroids compared to the $1 \mathrm{~g}$ control group. f. Gene expression of the tenocyte-specific transcription factor scleraxis $(S C X)$ was significantly increased in spheroids compared to the control group (3.7x, $\mathrm{SD}=0.69 \mathrm{p}=0.03)$.

loss of somatic cell has been reported in monolayer culture. Chondrocytes showed loss of chondrogenic gene expression (Sox-9, c-fos, and c-jun) ${ }^{23}$. Furthermore renal mesangial cells show phenotype loss during the first five passages ${ }^{24}$. As known from our own prior experiments ${ }^{25}$ and investigated by $\mathrm{Yao}$ et al. in detail $^{4}$, dedifferentiation of human tenocytes occurs after early passaging. Three-dimensional culture models have been shown to counteract in vitro dedifferentiation to a certain extent. 3D-culture models can be divided into those utilizing scaffold materials and scaffold-free, cell-only systems. Scaffold free-systems, such as hanging drop spheroid formation, have been shown to better preserve regenerative capacity of mesenchymal stem cells compared to monolayer culture $^{26}$. Embedding in scaffold materials such as 
PLGA has been shown to preserve tenocyte phenotype better than 2D culture ${ }^{8}$. However, cell expansion and passaging out of scaffold materials is laboursome and not suitable for routine purposes. With simulated microgravity, we propose a 3D culture model that has several advantages: it is a cell-only environment that avoids problems known from scaffold systems, such as difficulties in harvesting cells. Furthermore, it is not limited in size and dimension, such as the hanging drop system, and it is not limited in its duration of application. No impaction of cell viability was observed in our experiments during the full observation period. Semiquantitative observation in this study revealed a cell layer close to confluence in the control group and in the experimental group, so that at least no obvious deceleration of cell growth occurred. Potential differences in cell proliferation will be quantified in future experiments. We furthermore observed higher expression of genes that reflect tenogenic phenotype, such as collagen 1 , collagen 3 and scleraxis. This occured particularly in the spheroids, which may indicate better phenotype preservation than in 2D culture, in analogy to other 3D culture models. There was a tendency of increased gene expression for tenascin c, fibronectin and vimentin in the adherent cells under microgravity, but without significance. Upregulation of these cytoskeleton genes in the adherent cells rather than in the spheroids seems to follow some logic, as initial cell attachment under low gravity might require higher expression of cytoskeleton genes. Lack of significance in these results may be either due to an experimental group number too low or due to a measurement time point that was either too early or too late to detect maximum expression in these genes. Especially cytoskeleton is a cell compound that has been shown to react very sensitively on gravity alterations. For chondrocytes, perinuclear accumulation of the cytoskeletal component $\beta$-tubulin was detected after 16 hours in $\mathrm{RPM}^{27}$. Our further experiments will evaluate effects of microgravity on tenocyte cytoskeleton in more detail. Tenocytes have been shown to react very sensitively on force excertion. Cyclic strain has been shown to induce collagen production, proliferation and cytoskeleton alignment in rabbit tenocytes ${ }^{28}$. Reduction of forces under microgravity promises to have various effects on tenocyte cytoskeleton that are still to be evaluated. The question to what extent tenocyte spheroids may withstand mechanical forces and gain a shape that would allow them to serve directly as a tendon replacement is still to be elucidated. Many researchers and clinicians would appreciate a scaffoldfree tissue engineered tendon construct due to reasons of costs and biocompatibility. Microgravity has been shown to have beneficial effects on the cultivation of various other cell types before. For human chondrocytes, better preservation of collagen II production of the time under microgravity has been described $^{27}$, although effects of microgravity on cell function are various. The ultimate evidence that cancer cells lose their malignancy under microgravity is still missing, but there are some clues in this direc- tion. Lung cancer stem cells have been observed to lose their stemness under microgravity, downregulating Oct-4 and Nanog expression ${ }^{29}$. Thyroid cancer cells have shown higher production of antiproliferative and antimetastatic factors ${ }^{30}$.

\section{Conclusion}

We have proposed a 3D culture model that is easy to use and may help to preserve tenocyte phenotype better than conventional 2D culture. This might facilitate tenocyte cultivation for tissue engineering purposes in the future. With further long-term expansion of tenocyte spheroids, this could also be a step towards scaffold-free tissue engineering of a tendon construct. Future experiment will evaluate these issues in detail, particularly with respect to size, shape and mechanical stability.

\section{Authors' role/participation}

Armin Kraus: study design, conduction of experiments, writing of the manuscript.

Ronald Luetzenberg: study design, review of manuscript.

Nauras Abuagela: conduction of experiments, cell culture maintenance, review of manuscript.

Siri Hollenberg: review of manuscript, study design.

Manfred Infanger: study design, sponsorship, review of manuscript.

\section{References}

1. Nowotny J, Aibibu D, Farack J, Nimtschke U, Hild M, Gelinsky $M$, et al. Novel fiber-based pure chitosan scaffold for tendon augmentation: biomechanical and cell biological evaluation. $J$ Biomater Sci Polym Ed. 2016:1-20.

2. Pridgen BC, Woon CY, Kim M, Thorfinn J, Lindsey D, Pham H, et al. Flexor tendon tissue engineering: acellularization of human flexor tendons with preservation of biomechanical properties and biocompatibility. Tissue Eng Part C Methods. 2011;17(8):819-828.

3. Vaquette C, Slimani S, Kahn CJ, Tran N, Rahouadj R, Wang $X$. A poly (lactic-co-glycolic acid) knitted scaffold for tendon tissue engineering: an in vitro and in vivo study. J Biomater Sci Polym Ed. 2010;21(13):1737-1760.

4. Yao D, Liu H, Fan Y. Silk scaffolds for musculoskeletal tissue engineering. Exp Biol Med (Maywood). 2016;241(3):238-245.

5. Deng D, Liu W, Xu F, Yang Y, Zhou G, Zhang WJ, et al. Engineering human neo-tendon tissue in vitro with human dermal fibroblasts under static mechanical strain. Biomaterials. 2009;30(35):6724-6730.

6. Woon CY, Farnebo S, Schmitt T, Kraus A, Megerle K, Pham $\mathrm{H}$, et al. Human flexor tendon tissue engineering: revitalization of biostatic allograft scaffolds. Tissue Eng Part A. 2012;18(2324):2406-2417.

7. Whitlock PW, Seyler TM, Northam CN, Smith TL, Poehling GG, Koman LA, et al. Effect of cyclic strain on tensile properties of a naturally derived, decellularized tendon scaffold seeded with allogeneic tenocytes and associated messenger RNA expression. J Surg Orthop Adv. 2013;22(3):224-232. 
8. Stoll C, John T, Endres M, Rosen C, Kaps C, Kohl B, et al. Extracellular matrix expression of human tenocytes in three-dimensional air-liquid and PLGA cultures compared with tendon tissue: implications for tendon tissue engineering. J Orthop Res. 2010;28(9):1170-1177.

9. Riwaldt S, Bauer J, Wehland M, Slumstrup L, Kopp S, Warnke E, et al. Pathways Regulating Spheroid Formation of Human Follicular Thyroid Cancer Cells under Simulated Microgravity Conditions: A Genetic Approach. Int J Mol Sci. 2016;17(4).

10. Li H, Dai Y, Shu J, Yu R, Guo Y, Chen J. Spheroid cultures promote the stemness of corneal stromal cells. Tissue Cell. 2015;47(1):39-48.

11. Infanger M, Kossmehl P, Shakibaei M, Baatout S, Witzing A, Grosse J, et al. Induction of three-dimensional assembly and increase in apoptosis of human endothelial cells by simulated microgravity: impact of vascular endothelial growth factor. Apoptosis. 2006;11(5):749-764.

12. Padulo J, Oliva F, Frizziero A, Maffulli N. Muscles, Ligaments and Tendons Journal - Basic principles and recommendations in clinical and field Science Research: 2016 Update. Muscles Ligaments Tendons J. 2016;6(1):1-5.

13. Wuest SL, Richard S, Kopp S, Grimm D, Egli M. Simulated microgravity: critical review on the use of random positioning machines for mammalian cell culture. Biomed Res Int. 2015;2015:971474.

14. Stamenkovic V, Keller G, Nesic D, Cogoli A, Grogan SP. Neocartilage formation in $1 \mathrm{~g}$, simulated, and microgravity environments: implications for tissue engineering. Tissue Eng Part A. 2010;16(5):1729-36.

15. Pietsch J, Ma X, Wehland M, Aleshcheva G, Schwarzwalder A, Segerer J, et al. Spheroid formation of human thyroid cancer cells in an automated culturing system during the Shenzhou-8 Space mission. Biomaterials. 2013;34(31):7694-705.

16. Fulzele $S$, Arounleut $P$, Cain $M$, Herberg $S$, Hunter $M$, Wenger $\mathrm{K}$, et al. Role of myostatin (GDF-8) signaling in the human anterior cruciate ligament. J Orthop Res. 2010;28(8):1113-8.

17. Liu M, Yang J, Xing X, Cui X, Li M. Interleukin-17A promotes functional activation of systemic sclerosis patient-derived dermal vascular smooth muscle cells by extracellular-regulated protein kinases signalling pathway. Arthritis Res Ther. 2014; 16(6):4223.

18. Han S, Sidell N, Roser-Page S, Roman J. Fibronectin stimulates human lung carcinoma cell growth by inducing cyclooxygenase-2 (COX-2) expression. Int J Cancer. 2004;111(3):32231.

19. Kim H, Choi JA, Kim JH. Ras promotes transforming growth factor-beta (TGF-beta)-induced epithelial-mesenchymal transition via a leukotriene B4 receptor-2-linked cascade in mammary epithelial cells. J Biol Chem. 2014;289(32):22151-60.

20. Wall ME, Bernacki SH, Loboa EG. Effects of serial passaging on the adipogenic and osteogenic differentiation potential of adipose-derived human mesenchymal stem cells. Tissue Eng. 2007;13(6):1291-8.

21. Di Battista JA, Shebaby W, Kizilay O, Hamade E, Abou Merhi $\mathrm{R}$, Mebarek $\mathrm{S}$, et al. Proliferation and differentiation of human adipose-derived mesenchymal stem cells (ASCs) into osteoblastic lineage are passage dependent. Inflamm Res. 2014;63(11):907-17.

22. Lu HF, Narayanan K, Lim SX, Gao S, Leong MF, Wan AC. A 3D microfibrous scaffold for long-term human pluripotent stem cell self-renewal under chemically defined conditions. Biomaterials. 2012;33(8):2419-30.

23. Lin Z, Fitzgerald JB, Xu J, Willers C, Wood D, Grodzinsky AJ, et al. Gene expression profiles of human chondrocytes during passaged monolayer cultivation. J Orthop Res. 2008;26(9): 1230-7.

24. Ouardani M, Travo P, Bastie MJ, Mornet D, Neff S, LeungTack J. Loss of differences in mesangial cell phenotype between diabetic and normal rats: role of culture passages. Biol Cell. 1996;86(2-3):127-33.

25. Kraus A, Woon C, Raghavan S, Megerle K, Pham H, Chang J. Co-culture of human adipose-derived stem cells with tenocytes increases proliferation and induces differentiation into a tenogenic lineage. Plast Reconstr Surg. 2013;132(5):754e-66e.

26. Xu Y, Shi T, Xu A, Zhang L. 3D spheroid culture enhances survival and therapeutic capacities of MSCs injected into ischemic kidney. J Cell Mol Med. 2016.

27. Aleshcheva G, Sahana J, Ma X, Hauslage J, Hemmersbach $\mathrm{R}$, Egli M, et al. Changes in morphology, gene expression and protein content in chondrocytes cultured on a random positioning machine. PLoS One. 2013;8(11):e79057.

28. Riboh J, Chong AK, Pham H, Longaker M, Jacobs C, Chang J. Optimization of flexor tendon tissue engineering with a cyclic strain bioreactor. J Hand Surg Am. 2008;33(8):1388-96.

29. Pisanu ME, Noto A, De Vitis C, Masiello MG, Coluccia P, Proietti S, et al. Lung cancer stem cell lose their stemness default state after exposure to microgravity. Biomed Res Int. 2014;2014:470253.

30. Ma X, Pietsch J, Wehland M, Schulz H, Saar K, Hubner N, et al. Differential gene expression profile and altered cytokine secretion of thyroid cancer cells in space. FASEB J. 2014;28 (2):813-35. 